\title{
Transmission and Reception Tests of Digital Terrestrial TV in the Metropolitan Region of Curitiba
}

\author{
Célio Lúcio Vasco, José Frederico Rehme, Ruth Angelina Martins, Elias Agostinho, Alexandre de \\ Almeida Prado Pohl, Richard Demo de Souza, Keiko Verônica Ono Fonseca, and Marcelo Eduardo \\ Pellenz
}

\begin{abstract}
This paper describes the preliminary test results of the transmission and reception of Digital TV signals, on Channel 41, in the metropolitan region of Curitiba, in the state of Paraná, Brazil. It describes the equipment configuration deployed to carry out the tests, with the objective of mapping signal levels in the field and carrying out objective (channel power received, $\mathrm{C} / \mathrm{N}, \mathrm{MER}$ ) and subjective measures (signal quality and observation of artifacts) in 200 selected locations in the metropolitan region of Curitiba. A propagation model is discussed (based on the Log-distance and Okumura-Hata models), as a function of the received channel power values measured in some selected locations. The results should allow the calculation of the coverage area, and the identification of the shadowed areas and critical reception points.
\end{abstract}

Index Terms-

\section{INTRODUCTION}

$\mathrm{T}$ HE standard for digital TV in Brazil is set by a regulatory framework in the country, as well as increasing activity in the broadcasting area. With the introduction of new standards in audio and video encoding, middleware and transmission of RF signals, intensified research, project implementation and consequently trade in this area, including new receivers.

The definition of the Brazilian Digital Television Terrestrial System - SBTVD-T was determined by Decree No. 5820 [1] on 06/29/2006, which established the Japanese system ISDB$\mathrm{T}$ (Integrated Services Digital Broadcasting Terrestrial) as the

Célio Lúcio Vasco, Universidade Tecnológica Federal do Paraná (UTFPR) (e-mail: celiov@yahoo.com.br)

José Frederico Rehme, Rede Paranaense de Comunicação (RPC) (e-mail: fredrehme@gmail.com)

Ruth Angelina Martins, Rede Paranaense de Comunicação (RPC) (e-mail: ruthm@tvparanaense.tv.br)

Elias Agostinho, Associação das Emissoras de Radiodifusão do Paraná (AERP) (e-mail: elias@sertpr.org.br)

Alexandre de Almeida Prado Pohl, Universidade Tecnológica Federal do Paraná (UTFPR) (e-mail: pohl@cpgei.cefetpr.br)

Richard Demo de Souza, Universidade Tecnológica Federal do Paraná (UTFPR) (e-mail: richard@cpgei.cefetpr.br)

Keiko Verônica Ono Fonseca, Universidade Tecnológica Federal do Paraná (UTFPR) (e-mail: keiko@utfpr.edu.br)

Marcelo Eduardo Pellenz, Pontificia Universidade Católica do Paraná (PUC-PR) (e-mail: marcelo@ppgia.pucpr.br) transmission standard and also a transitional period of ten years for the transition from analog and digital. The official start of digital TV transmissions in Brazil [2] took place on December 2, 2007, when it began broadcasting in the city of São Paulo, since followed by other state capitals in the country.

One of the challenges that this new technology brings is to determine if the coverage of the digital signal will have the same range as analog broadcasting since the cost benefit between quality and robustness of the signal can cause differences in the signal coverage area. Coupled with the fact that with digital receivers, images can freeze when the reception level meets the reception threshold, unlike analog receivers.

With this in focus, this paper describes the preliminary results of the transmission and reception tests for a digital TV channel in the metropolitan region of Curitiba, the state capital of Paraná, the focus is the determination of an empirical propagation model that will agree with the measurements taken.

The purpose of surveying the digital TV broadcast signals in Curitiba is to know the propagation characteristics within the region of the new modulated digital signals to determine if there are critical points or shadow areas. This study is of interest to all broadcasters wishing to deploy digital TV systems in the region.

The tests are being conducted in a partnership between the Universidade Tecnológica Federal do Paraná (UTFPR) and the Associação das Emissoras de Radiodifusão do Paraná (AERP), with assistance from Rede Paranaense de Communicação (RPC).

This article describes the infrastructure used for the survey, the propagation models used, the analysis of the collected data, the estimated signal coverage and conclusions.

\section{INFRASTRUCTURE}

The early trials of Digital TV transmission in Curitiba took place with the authorization for the UTFPR to run the Special Service for scientific or experimental purposes conforming to Act no. 1388 [3] on 03/12/2008, issued by the Agência Nacional de Telecommunicações (Anatel).

The feasibility study was made possible by RPC, one of the main Paranaense broadcasters associated with AERP, which 
provided the necessary infrastructure, i.e., the transmitter and the radiant system installed on a tower, operating on UHF channel 41. The survey began in June 2008 and is still ongoing during the period of this article being written. Therefore, the results discussed in this paper are only partial.

\section{A. Transmission}

The transmission system characteristics are described in Act no. 1388 issued by Anatel [3], as follows:

a) The transmitter equipment used is manufactured by Linear Electronic Equipment SA, Model IS74K1 with power operation up to $4.1 \mathrm{~kW}$ tuned to channel 41 , occupying the range of frequencies between $632-638 \mathrm{MHz}$;

b) The radiant system used is manufactured by Transtel Conti \& Cia Ltda., model TTSL8 U0 - 41-10D, omnidirectional, with 8 slots, with a total gain of $10.89 \mathrm{dBd}$;

c) The geometric center of the radiating system is installed $90.0 \mathrm{~m}$ above the quota basis, $950 \mathrm{~m}$ above sea level. The azimuth orientation is $0^{\circ} \mathrm{NV}$.

The transmitter was tested in five different configurations, for the configuration levels analyzed the transmission power is $4.1 \mathrm{~kW}$, the modulation is 64QAM, the guard interval is $1 / 16$ and FEC rate is $3 / 4$. Video content was transmitted in a sequence of different scenes, which were non-commercial.

\section{B. Reception}

The collection of reception data is being completed in a vehicle called the mobile field measurement unit from RPC, equipped with:

a) an $8 \mathrm{~m}$ telescopic mast, equipped with a log-periodic antenna manufactured by Proeletronic with full UHF band for the reception of channel 41. It was also equipped with an antenna for the reception and evaluation of analog signals from channel 12, not forming part this study;

b) a manufacturer's spectrum analyzer, Agilent ESA model E $4405 \mathrm{~B}$ for measuring the reception power;

c) a digital television receiver connected to a display with the aspect ratio of 16: 9. This allows the subjective quality scoring of the received signal;

d) a laptop for data collection;

e) a GPS receiver for measuring location coordinates;

f) A thermohygrometer for measuring temperature and humidity;

The height of an $8 \mathrm{~m}$ mast antenna on the mobile unit, as seen in Fig. 1 allows the simulation of a residential receiving antenna. Fig. 2 shows the inside of the mobile unit, where the equipment layout can be seen.

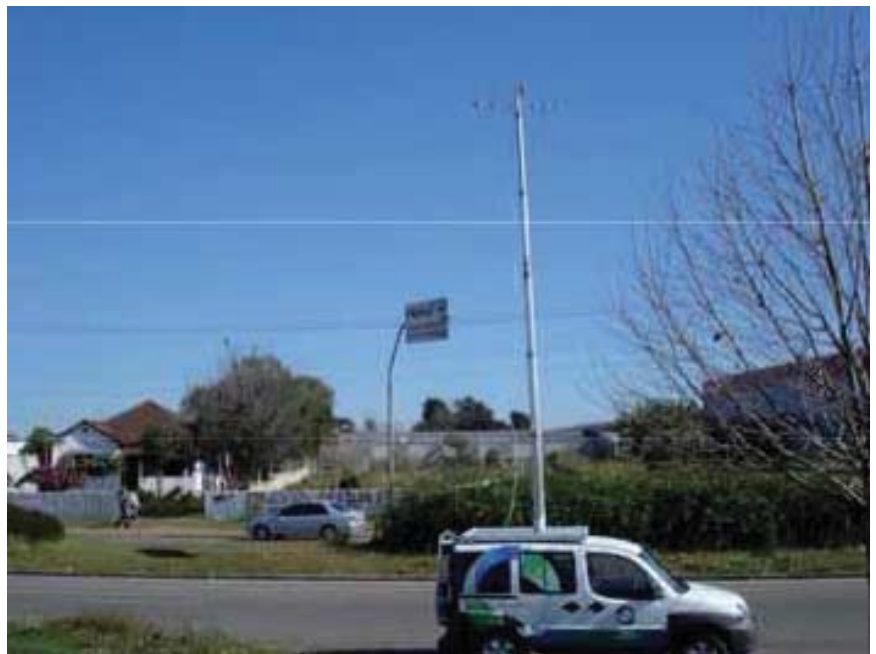

Fig. 1. Mobile field measurement unit

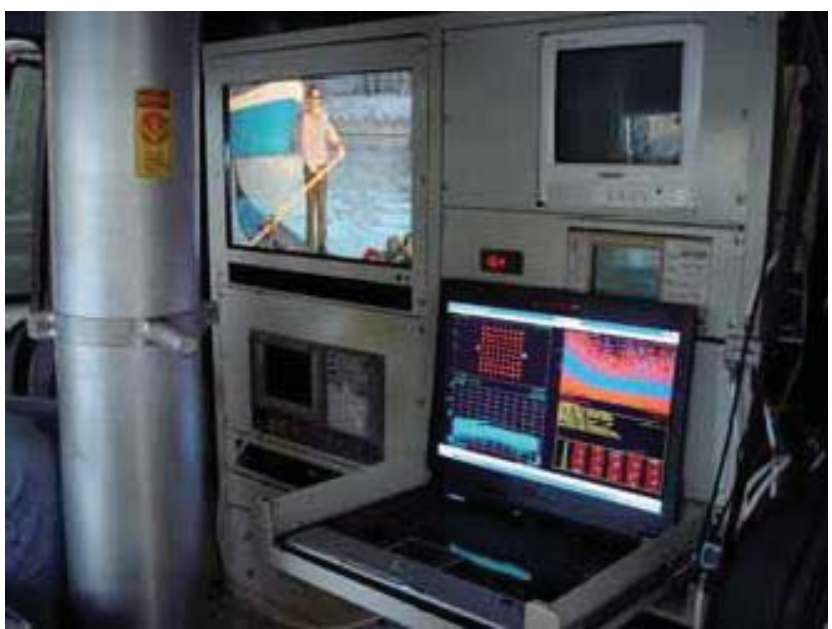

Fig. 2. Interior of the mobile unit

\section{DATA ANALYSIS}

The database considered consists of the measurements collected in 87 locations assessed from June 13 until July 17, distributed around the city of Curitiba.

In this study only the $6 \mathrm{MHz}$ bandwidth channel power was analyzed, measured at the receiver, with the transmission at $4.1 \mathrm{~kW}$, broadcasting in high definition, which allows evaluation of the propagation characteristics in the region of Curitiba in normal operating conditions.

\section{A. Propagation model choice}

The initial comparison of the receiving power measurement was with the free space propagation model, which enables the prediction of signal attenuation in an unobstructed line of sight between the transmitter and the receiver.

Using Friis' free space equation, described by Rappaport [4], the reception power $P_{r}$ is given by:

$P_{r}(d)=\frac{P_{t} G_{t} G_{r} \lambda^{2}}{(4 \pi)^{2} d^{2} L}$

where $P_{t}$ is the transmitter power, $G_{t}$ is the gain of the transmitting antenna, $G_{r}$ is the gain of the receiving antenna, $\lambda$ is the wavelength of the transmitted signal, $d$ is the distance 
between the transmitter and receiver, and $L$ is other losses not related to the propagation.

This model considers the transmitter power, the antenna gain, and operating frequency, as the received power decreases with the square mean of the distance. In the results from the implementation of this model, shown in Fig. 3, it is observed that the position of the curve is well above the plotted locations, indicating that this prediction model is far from the real situation.

As the received signal level decreases logarithmically with the distance, the Log-distance path loss model was used, as described by Rappaport [4] and is calculated by:

$\operatorname{PL}(d)=\operatorname{PL}\left(d_{0}\right)+10 n \log \left(d / d_{0}\right)$

where PL is the propagation loss between the distances of $d$ and $d_{0}$, where: $d$ is the distance from the transmitter to the measured location, $d_{0}$ is the distance to the nearest transmitter reference, and $n$ is the propagation loss exponent. In the evaluation, it was considered that $d_{0}$ is equal to $200 \mathrm{~m}$, that is, the distance from the transmitter to the measured location nearest to the antenna and $\operatorname{PL}\left(d_{0}\right)$ the average propagation loss at the measured locations to $200 \mathrm{~m}$.

The exponent $n$ of the Log-distance model was obtained using the minimum-mean-square-error method (MMSE), where $n=2.8267$ is obtained, as shown in Fig. 3. This value indicates that the decay of the reception level is greater than in the in the case of free space attenuation, or that the environment causes more severe losses than in free space conditions. According to Rappaport [4], $2.7<n<3.5$ indicates an environment in urban areas, consistent with the calculated value. To analyze the dispersion values around the mean, the standard deviation was calculated, and as shown in Fig. 3 the value found was $\sigma=10.1 \mathrm{~dB}$, indicating that the reception power may have considerable variation around the mean.

This normalization logarithmic model in which the propagation loss is characterized by an attenuation factor, which, in this case, is the distance exponent $n$ is a nonfrequency dependent model that can be used in various transmission bands, and the value of $n$ intrinsically contains the effect of all propagation mechanisms [5].

The Hata model [6] is a mathematical formula for empirical graphic base of attenuation losses provided by Okumura, and valid for the range of $150 \mathrm{MHz}$ to $1500 \mathrm{MHz}$, this was implemented as described by Rappaport [4] and is calculated by:

$$
\begin{gathered}
L_{50}=59.55+26.16 \log f_{c}-13.82 \log h_{t e} \\
-\mathrm{a}\left(h_{r e}\right)+\left(44.9-6.55 \log h_{t e}\right) \log d
\end{gathered}
$$

where $\mathrm{L}_{50}$ is the propagation loss in $\mathrm{dB} ; f_{c}$ is the operating frequency; $h_{r e}$ is the height of the transmission antenna, $h_{r e}$ is the height of the receiving antenna, and $d$ is the distance from transmitter to receiver. The correction factor for a large city and frequency greater than $300 \mathrm{MHz}$, is given by:

$a\left(h_{r e}\right)=3.2\left(\log 11.75 h_{r e}\right)^{2}-4.97$.

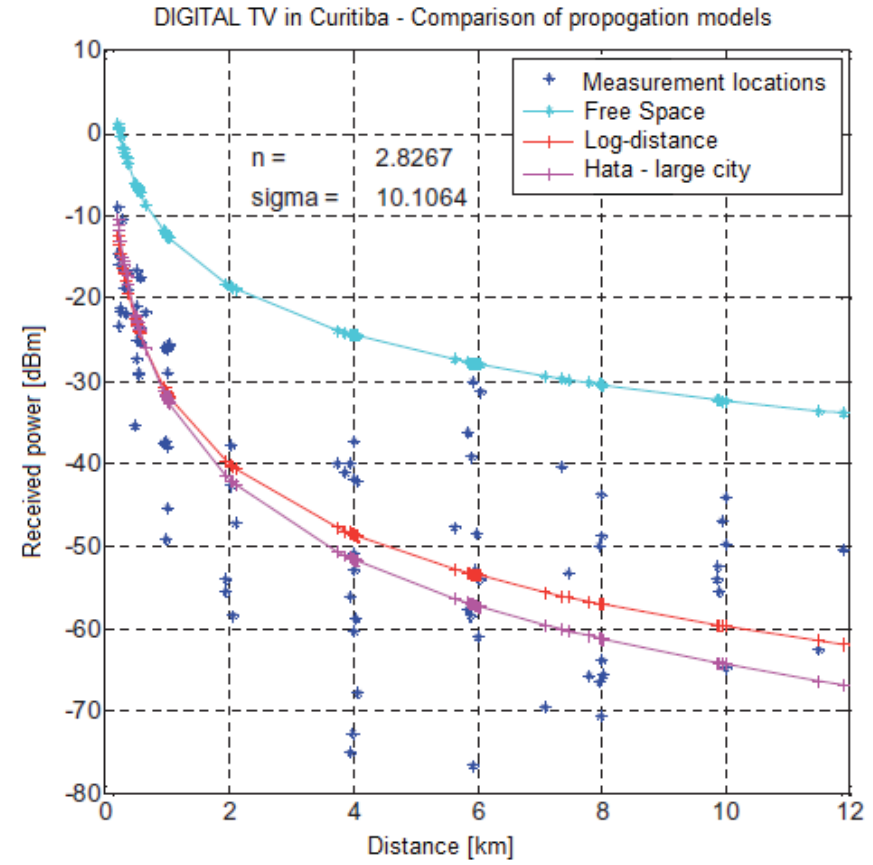

Fig. 3. Analysis of reception power for the free space, Log-distance, and Hata models

\section{B. Improving the estimation of received power}

The evaluation of the reception power data indicates that the Log-distance and Hata models can be adopted by Curitiba, but it is observed that the standard deviation between the measured values were compared with the average estimated at $10.1 \mathrm{~dB}$, a value that would be wanted to be reduced. Therefore, we investigated a way to have a more detailed estimate of the estimated channel power values received at the considered locations.

In an attempt to minimize the standard deviation, stratification of the received power values was completed in three sets in relation to the average: a) within $\pm 10 \mathrm{~dB}, \mathrm{~b}$ ) above $10 \mathrm{~dB}$ c) below $10 \mathrm{~dB}$. Fig. 4 presents this stratification. 


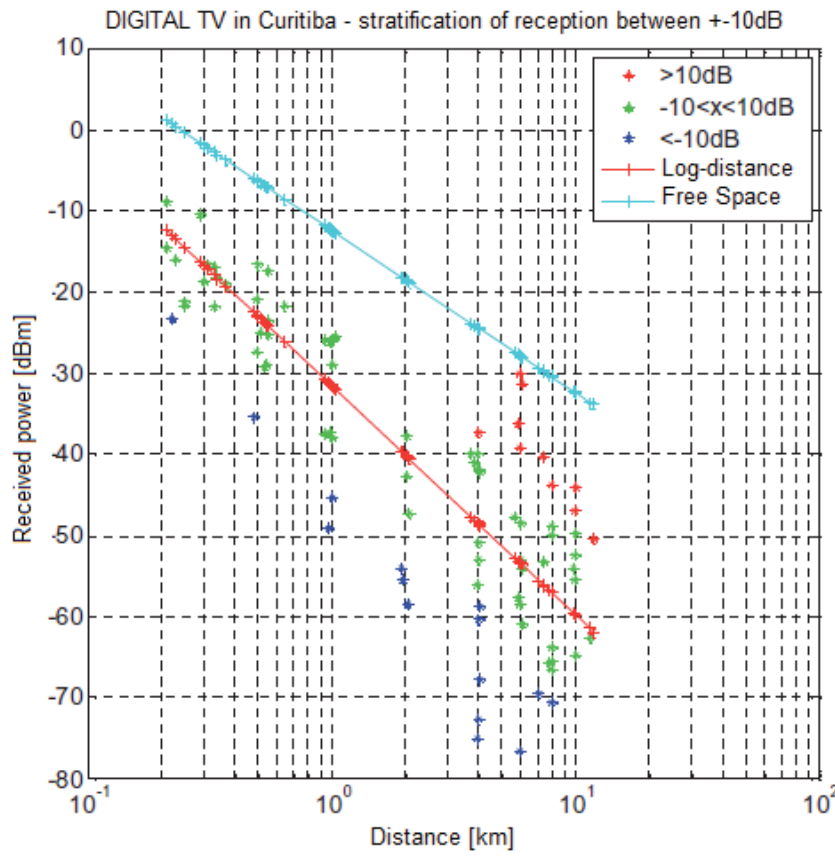

Fig. 4. Stratification of the received power within the standard deviation

It is observed that there are ten values above the standard deviation limit and fifteen values below the standard deviation limit. An interpretation of the values below the limit is the existence of other signal attenuations that have not been considered, such as, for example, losses due to obstruction. The values above the variance should indicate a line of sight situation between the receiver and the transmitter, approaching the free space model.

\section{Obstruction by attenuation analysis}

As the city of Curitiba does not have uniform topography, and because of the existence of concentrations of buildings along the line of sight of transmissions, a situatio typical of big cities, attenuation by obstruction was analyzed due to the signal diffraction phenomenon. This phenomenon is present when obstacles, such as hills blocking line of sight signals, causing attenuation that adds to the attenuation of free space propagation [7].

From terrain profile data obtained from the SIGAnatel system, it was possible to make a survey of Curitiba, shown in Fig. 5, with a radius of $12 \mathrm{~km}$, and with a precision of $90 \mathrm{~m}$. The Sistema de Informações Geográficas Anatel (SIGAnatel) [8] is an application available on the website of the National Telecommunications Agency (Anatel), which contains geographical and topographical data of Brazil, and enables a propagation analysis module.

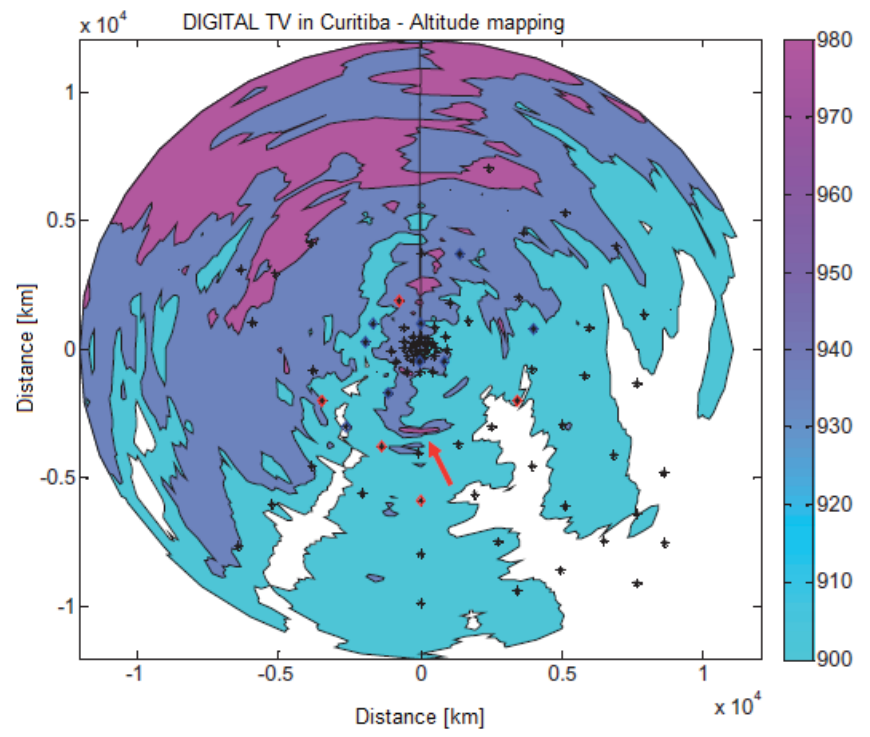

Fig. 5. Topographic map of Curitiba

It is observed that the transmitting antenna in the center of Fig. 5, is situated at a median altitude of the region, specifically $950 \mathrm{~m}$. To the north, there is a higher region and to the southeast there is a lower region. Approximately $3 \mathrm{~km}$ south of the antenna, there is a region with a high concentration of buildings that are the same height as the atenna's base obstructing line of sight transmissions to the southern region.

In the map in Fig. 5, the altitude of some regions of Curitiba, which were considered relevant to the survey, those $60 \mathrm{~m}$ above ground level mark the height of buildings. These regions were obtained through empirical analysis using GoogleEarth and are shown in Fig. 6. The marked areas indicate regions with concentrations of buildings, and the altitude of the region indicated by the arrow in the south azimuth has the same dimensions as the transmission antenna's base.

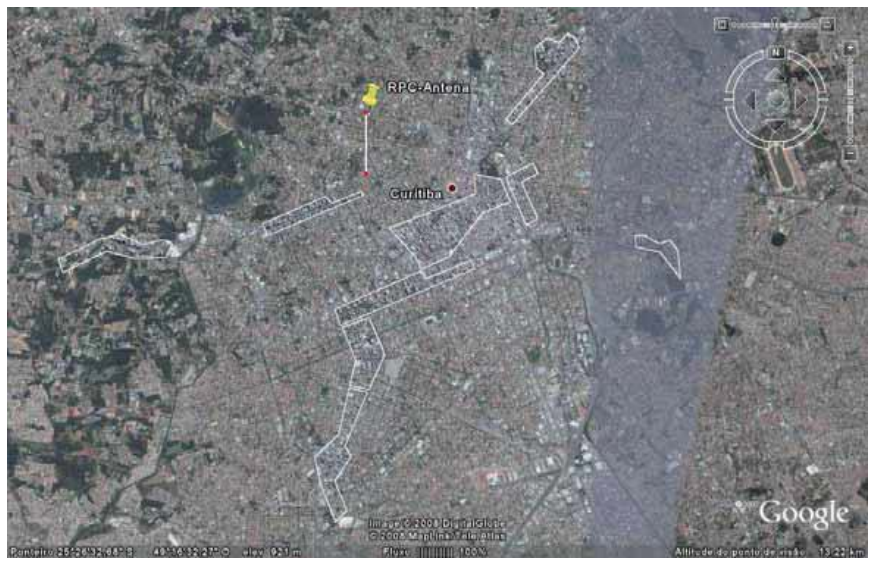

Fig. 6. Areas with high concentrations of buildings

From the terrain profile database and the marking of buildings, mapping of the loss due to obstruction in the city was completed, for each of the azimuths from $0^{\circ}$ to $350^{\circ}$, in steps of $10^{\circ}$, and a variation of distance from $1 \mathrm{~km}$ to $12 \mathrm{~km}$ in 
$90 \mathrm{~m}$ steps. The $v$ parameter from Fresnel-Kirchoff's diffraction was used, as described by Rappaport [4], and is calculated by:

$v=h \sqrt{\frac{2\left(d_{1}+d_{2}\right)}{\lambda d_{1} d_{2}}}$

where $v$ is the Fresnel-Kirchoff diffraction parameter, onedimensional, $\lambda$ is the signal wavelength transmitted, $h$ is the obstruction height above the line of sight between the transmitting antenna and the receiving antenna, $d_{1}$ is the distance between the transmitting antenna and obstruction, and $d_{2}$ is the distance between the obstruction and the receiving antenna.

From the $v$ diffraction parameter, the obstruction loss $P_{d}$ in $\mathrm{dB}$ was calculated, as described by Rappaport [4] and is given by:

$$
\begin{gathered}
P_{d}=-20 \log (0.5 \exp (-0.95 v)) \quad 0 \leq v \leq 1 \\
P_{d}=-20 \log \left(0.4 \sqrt{0.1184-(0.38-0.1 v)^{2}}\right)
\end{gathered}
$$

$1 \leq v \leq 2.4$

$P_{d}=-20 \log \left(\frac{0.225}{v}\right)$

$$
v>2.4
$$

The mapping of the loss by obstruction is shown in Fig. 7.

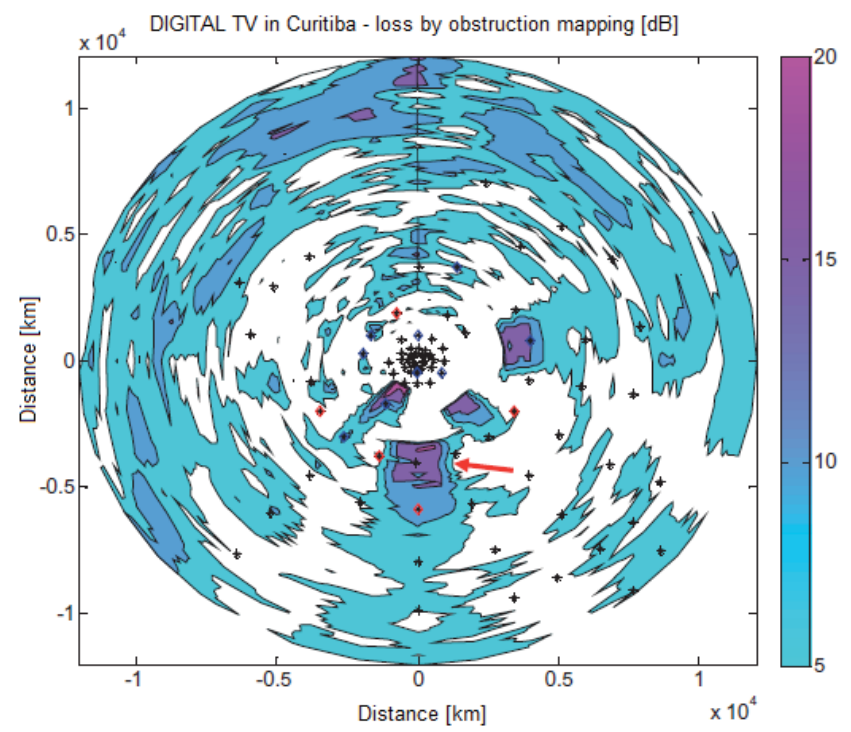

Fig. 7. Map of signal loss caused by obstructions in Curitiba

Of the 87 locations examined, 49 locations were found to have an attenuation loss greater than $0 \mathrm{~dB}$, with an average of $5.6 \mathrm{~dB}$ and a standard deviation of $3.5 \mathrm{~dB}$. The maximum attenuation of $16.7 \mathrm{~dB}$ was found in azimuth $181^{\circ}$ at a distance of $4 \mathrm{~km}$ (region marked by the arrow), followed by $15.2 \mathrm{~dB}$ at $79^{\circ}$ and $4 \mathrm{~km}$, and $12.6 \mathrm{~dB}$ at $180^{\circ}$ and $5.9 \mathrm{~km}$. These points are in agreement with those regions that were pointed out in Fig. 6.

It is observed in Fig. 7 that there are shadow areas caused by the density of buildings in some regions of the city, which can cause a greater loss than $15 \mathrm{~dB}$ in signal strength. The arrow indicates the shadow region in the south azimuth caused by a cluster of buildings also marked in Fig. 6. The white regions indicate areas with obstruction loss less than $5 \mathrm{~dB}$ and must have lines of sight between the transmitter and the receiver, or is unobstructed.

\section{Propagation model analysis considering loss by obstruction}

With the calculation of loss by obstruction in the evaluated locations, the analysis was repeated with propagation models, and the results are presented in Fig. 8.

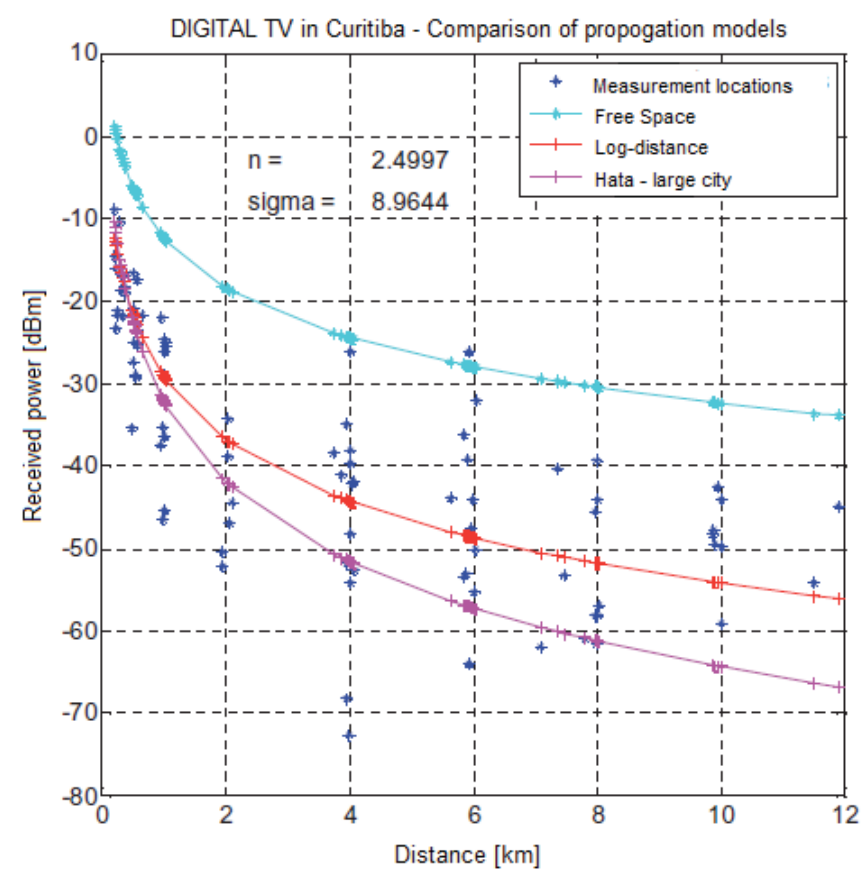

Fig. 8. Analysis of the received power, considering loss by obstruction, for the Free Space, Log-distance and Hata models

It was observed that there was an improvement of approximately $1 \mathrm{~dB}$ in the standard deviation of channel power values at the measured locations. The Log-distance curve indicates that the average scores are being less dispersed, i.e., these estimates are more precise. In relation to the stratification values, a new situation has been obtained. 


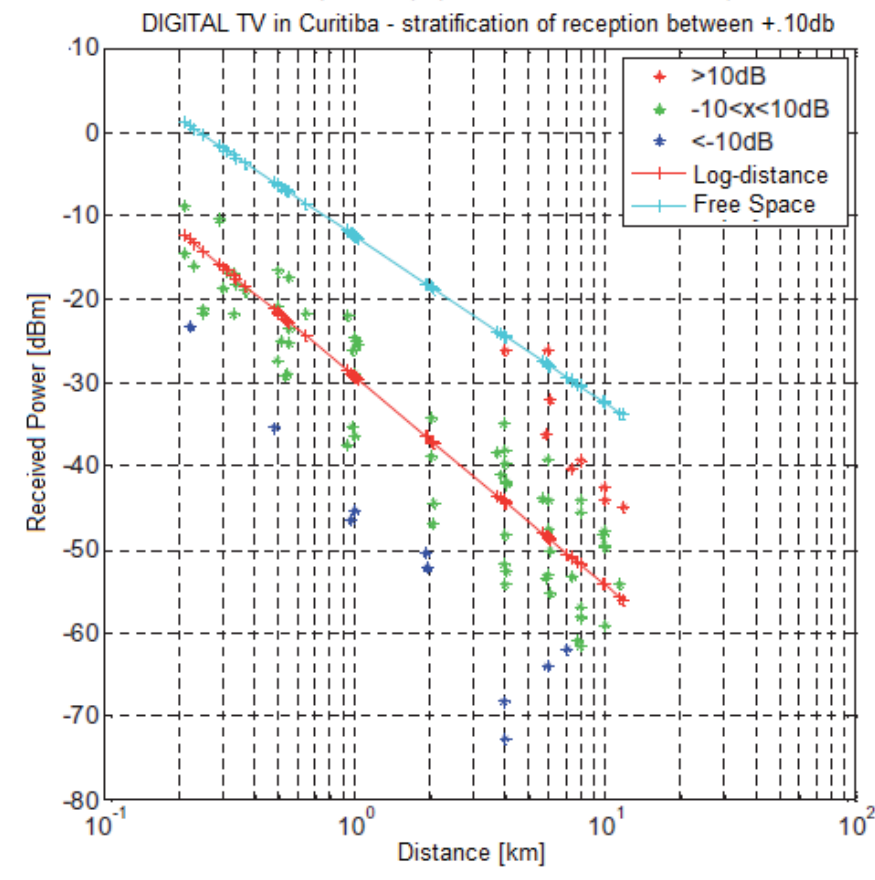

Fig. 9. Stratification of the powers received within the $\pm 10 \mathrm{~dB}$ deviation considering the loss by obstruction

It is observed that there are nine values above the standard deviation limit and ten below the standard deviation limit. There was an improvement in the dispersion of estimated values; however, there are still values that are outside the standard deviation. A location analysis is needed to check for possible loss by obstruction by local buildings, or other situations.

\section{Estimate of Signal Coverage}

After obtaining a suitable propagation model for the city of Curitiba, it is possible to estimate the Digital TV signal coverage distance to the parameters considered.

According to standard ABNT NBR 15604 [9] which regulates digital TV receivers, the receiver sensitivity must be less than $-77 \mathrm{dBm}$ at the receiver input.

Considering (2), the estimated Log-distance model in Section III.A, we have:

$\operatorname{PL}(d)=\operatorname{PL}\left(d_{4} ; \mathrm{E} \operatorname{srJ} \quad:_{4} \cdot @ \quad @\right.$

Therefore, considering the practical data: transmission power of $4.1 \mathrm{~kW}$, i.e., $66.12 \mathrm{dBm}$; reference power of $11.75 \mathrm{dBm}$ to $200 \mathrm{~m}$; minimum power reception of $-77.0 \mathrm{dBm}$; loss by propagation exponent of 2.8271; calculates the maximum distance of $d$ as $40.6 \mathrm{~km}$.

Concluding that, according to the estimate made in this work, from the empirical obtainment of a propagation loss curve, the digital TV signal coverage would have an average distance of $40.6 \mathrm{~km}$ for the region of Curitiba. Note that this estimate is in $50 \%$ of the cases, that is, values that are within the standard deviation from the evaluated locations and without a radial analysis, which could have different values.

To prove this estimate, a simulation of TV channel viability in the SIGAnatel system [8] was carried out with the same simulation data. This system has an application to simulate the coverage area of a digital TV channel, whereas the coverage area for the primary protection of transmission is the radius that the reception level threshold is $-51 \mathrm{~dB} \mu \mathrm{V}(-56 \mathrm{dBm})$. The result obtained in the SIGAnatel simulation, indicates that the average distance for the $-56 \mathrm{dBm}$ receiving power is $47.0 \mathrm{~km}$.

It is observed that the mean radius of the primary boundary for the $-51 \mathrm{~dB} \mu \mathrm{V}$ estimate generated by the SIGAnatel system is $47.0 \mathrm{~km}$, while the estimate according to the Log-distance curve field survey of reception power is $-77 \mathrm{dBm}$ and $40.6 \mathrm{~km}$. This difference may be because the analysis completed by the SIGAnatel system follows recommendation ITU-R 1546, which has a different propagation condition in relation to urban density.

In accordance with annex 7 of recommendation ITU-R P.1546-1 [10], the equation to obtain the field strength for the Okumura-Hata method is given by:

$$
\begin{aligned}
& \mathrm{L} x\left\{\mathrm{zt} F \mathrm{~F} \text { S } \mathrm{x} \text { su } \mathrm{zB} \mathrm{F}_{5}\right. \text { * } \\
& \mathrm{E}=: *_{6} ; \mathrm{F}: \mathrm{VV} \quad\left\{\mathrm{F} \times \quad \mathrm{W} \quad \mathrm{W}_{i}: \quad\right. \text { « ; }
\end{aligned}
$$

where $\mathrm{E}$ is the field strength in $\mathrm{dB} \mu \mathrm{V} / \mathrm{m}$ to $1 \mathrm{~kW}$ erp; $f$ is the operating frequency; $H_{1}$ is the height of the transmission antenna, $\mathrm{H}_{2}$ is the height of the receiving antenna, $d$ is the distance from the transmitter to the receiver, and $b$ is the correction factor dependent on $H_{1}$ and $\mathrm{d}$, where $\mathrm{b}=1$ for $\mathrm{d} \leq 20 \mathrm{~km}$. The correction factor for the receiving antenna is given by:

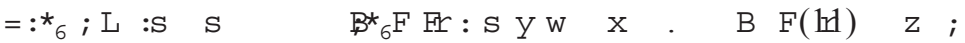

It is noted that in (11), the correction factor shown in recommendation ITU-R P.1546-1 is the same as that presented by Rappaport [4] and in (3.83), used for propagation in small and medium-sized cities. Therefore, it can be inferred that the model adopted by recommendation ITU-R P.1546-1 is equivalent to the Hata model for small and medium-sized cities.

In addition to the difference in density urban, according to recommendation no. 398 the coverage assessment is completed considering the height of the receiving antenna at $10 \mathrm{~m}$, which causes an increase in the level received at the receiver.

Therefore, the simulation completed by the SIGAnatel system considers a less rigid situation than observed empirically in Curitiba. Again, it is concluded that the estimated model for this work is suitable to be used in Curitiba.

\section{CONCLUSION}

The Log-distance propagation model is useful for providing an estimate from empirically collected data, the behavior of channel power in relation to distance. From this estimated model, we can also conclude that the Hata model used in urban areas of large cities, it is suitable for use in Curitiba.

In conclusion, according to the estimate made in this paper, the digital TV signal coverage for a city region would have a radius of $40.6 \mathrm{~km}$. Considering that this is a situation that will be needed in $50 \%$ of cases. 
There is a difference between the estimate obtained by this work and the estimate of coverage obtained by the SIGAnatel system, which adopts recommendation ITU-R P.1546-1 that considers average propagation conditions, ie, for small and medium-sized cities. While this work from local empirical data estimates that propagation conditions are more severe in the urban areas of large cities. This comparison will have a future breakdown.

This study, which is preliminary, may only be completed after the completion of data collection of the 200 locations it proposes in this research.

\section{REFERENCES}

[1] Presidência da República (2006). "Decreto n 5.820,” in Diário Oficial da União de 30/6/2006.

[2] Ministério das Comunicações (2007). "Ministro Hélio Costa fala sobre TV Digital." Available:

$<$ http://www.mc.gov.br/003/00301009.asp?ttCD CHAVE=19242>

[3] Anatel (2008). "Ato $n^{\circ} 1388$." in Diário Oficial da União, $n^{\circ} 51$, de $14 / 3 / 2008$.

[4] Rappaport, Theodore S. (1999). "Wireless Communications - Principles \& Practice," Prentice Hall, USA.

[5] C. Perez-Vega and J.M. Zamanillo, "Path-loss model for broadcasting applications and outdoor communication systems in the VHF and UHF bands." IEEE Trans. Broadcasting, vol. 46, no. 2, pp. 91-96, June. 2002.

[6] M. Hata, "Empirical Formula for Propagation Loss in Land Mobile Radio Services," IEEE Trans. Veh. Technol., vol.29, pp.317-325, Aug. 1980.

[7] Propagation by Diffraction, Recommendation ITU-R P.526-10, 2007.

[8] Anatel (2008). "SIGAnatel - Sistema de Informações Geográficas."

Available at: $\mathrm{http}: / /$ sistemas.anatel.gov.br/siganatel

[9] ABNT (2007). "ABNT NBR 15604 - Televisão digital terrestre Receptores," in Associação Brasileira de Normas Técnicas.

[10] Anatel (2005). "Rec. UIT-R P.1546-1 - Método de previsões ponto-área para serviços terrestres na faixa de freqüências de 30 a $3000 \mathrm{MHz}$ " in Anexo à Resolução Anatel n. ${ }^{\circ}$ 398, de 7/4/2005, Anexo II.

\footnotetext{
Cite this article:

Vasco,C.L., Rehme, J. F. , Martins, R. A., Agostinho, E., de Almeida Prado

Pohl, A., Demo de Souza, Richard, Ono Fonseca, K. V., Pellenz, M. E.;

2015.Transmission and Reception Tests of Digital Terrestrial TV in the

Metropolitan Region of Curitiba. SET INTERNATIONAL JOURNAL OF

BROADCAST ENGINEERING. ISSN Print: 2446-9246 ISSN Online:

2446-9432. doi: 10.18580/setijbe.2015.2. Web Link: http://

dx.doi.org/10.18580/setijbe.2015.2
} 